\title{
Study on Picture Book Creativity from the Perspective of the Inheritance of Intangible Cultural Heritage - Taking the Picture Book Design of Yancheng Huai Salt Drying Technology as an Example
}

\author{
Yanjun Wang ${ }^{1, *}$ Yajie Jiang ${ }^{1}$ \\ ${ }^{1}$ Xiamen Academy of Arts and Design, Fuzhou University, Xiamen, Fujian 361000, China \\ "Corresponding author. Email: 200885939@qq.com
}

\begin{abstract}
Purpose: The purpose of this paper is to explore the design performance and innovation of Huai salt drying techniques in picture book creativity. The current Huai salt technique is facing the practical problems of inheritance and development, and the way of spreading is expanded through picture book creativity. Method: Starting from the inheritance of Yancheng Huai salt art and culture, it is proposed that Huai salt art and culture can be used as a cultural theme for the creative design of picture books. This paper explains the development and application status of Yancheng Huai salt technology in picture book creativity, analyzes the importance of its application in picture book creativity, proposes to refer to and learn from excellent design cases, and concludes that it is necessary to reflect the practical application of Huai salt drying technology in picture book creativity from three aspects: creative ideas, fusion of various art forms, and design elements. Conclusion: Yancheng Huai salt drying technology is an intangible cultural heritage project that inherits traditional handicrafts. It carries the wisdom and wealth of salt people for thousands of years. On this basis, modern and diversified picture book design methods are used to make the public Be able to better understand the basic content of Yancheng Huai salt drying technology. Significance: It enriches the artistic methods and means of creative design of picture books, and at the same time effectively spreads the content and form of Yancheng Huai salt drying technology.
\end{abstract}

Keywords: Huai salt drying technology, Picture book creativity, Intangible cultural heritage.

\section{INTRODUCTION}

In recent years, China attaches great importance to the protection and inheritance of intangible cultural heritage, and has adopted many methods for communication. However, there are still many problems in the communication process, such as insufficient cultural expression, lack of innovation, etc. [1] With the progress and change of the times, Huai salt drying technology has also undergone different changes, but it stays roughly the same as before. The generations of inheritors of Huai salt technology still uphold the respect for the technology and pass on this technology with heart [2]. As one of the popular design trends in recent years, picture book creativity has the communication effect of combining pictures and texts, which is conducive to improving public acceptance and learning efficiency. This paper proposes to study Huai salt drying technology and techniques with the creativity of picture books, combining vision with a variety of art forms, using analogy methods to restore ancient techniques, and innovatively combining with Huai salt technology, so as to achieve the purpose of the inheritance of Huai salt drying technology and culture [3]. 


\section{THE DEVELOPMENT STATUS AND DESIGN IDEAS OF HUAI SALT DRYING TECHNOLOGY IN PICTURE BOOK CREATIVITY}

\subsection{Development Status}

\subsubsection{Development Process}

At present, there are relatively few picture books that mainly express the contents of Huai salt drying technology in picture book creativity. Most picture book ideas on the market are designed for the development and education of children, and the picture book ideas of traditional techniques are almost negligible. Through creative picture books to convey Huai salt drying technology that can more intuitively express the technology and content, add visual effects, tactile experience, and aesthetic feelings in the picture books, so that readers can have a deeper experience. Therefore, it is necessary to use Huai salt drying technology to do In-depth study of picture book design.

\subsubsection{Main Elements of Picture Book Creativity}

Picture book creativity is divided into four major elements: graphics, text, composition and book design. Graphics convey meaning, words express content, composition shows aesthetics, and book design highlights creativity. The performance of Huai salt drying technology needs to start with these four elements in order to form an organic whole. See "Figure 1".

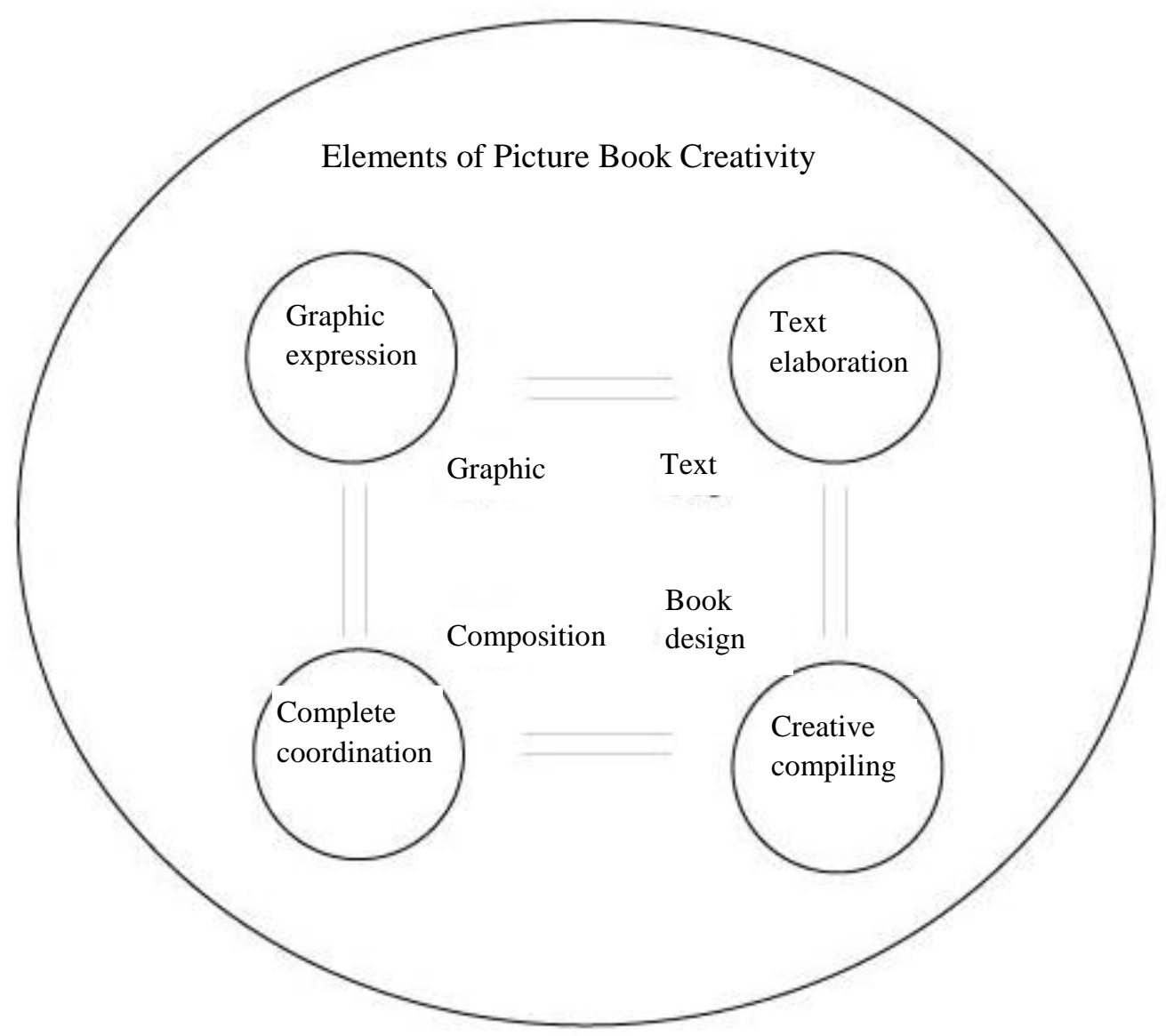

Figure 1 Creative elements of picture books (self-drawn).

\subsubsection{Graphics}

In picture book creativity, graphics are used to explain to readers through creative expressions according to certain textual intentions or express the image expression produced by the designer through thinking and subjective. It is a visual presentation that is contagious to the theme and is symbolic. Graphical expression is undoubtedly important to Huai salt drying technology, which can enhance the infectiousness of technical 
expression and improve the efficiency of understanding the technology.

\subsubsection{Text}

Like graphics, text also carries the function of describing content. The difference is that the narrative of the text is weak. Huai salt drying technology is a technique, it is intangible. It's far from enough to express through graphics alone, so a large amount of text is needed to support the expression of skills to stimulate the imagination of readers

\subsubsection{Composition}

The composition can unify the text and graphics into the same picture, forming a complete and coordinated picture, and increase the artistry. To a large extent, the novelty of the composition can determine the attractiveness of the picture. The composition of the Huai salt drying technology must follow all the principles of expressive skills, and arrange the pictures and texts reasonably to achieve the purpose of expressing skills.

\subsubsection{Book Design}

Book design is the most coordinated part of the entire picture book design. It can arrange relatively independent text and illustrations into a book, so that layout design, graphic arrangement, illustration depiction, cover design, opening and closing methods, etc. can be integrated, thus forming a complete creative picture book. Huai salt drying technology also needs to start from the aspects of graphic layout, layout design, cover design, opening and closing methods, etc., to artistically design the picture book to form an organic whole.

\subsubsection{Importance in Picture Book Creativity}

Picture book creativity is mainly to convey meaning through images and words, and image transmission can be more intuitive, allowing readers to more easily understand the main content of the craft. Picture books use traditional handicraft utensils as materials to create picture books, and use picture books as carriers to spread, protect, and develop traditional Chinese culture and craftsmanship. Adhering to cultural self-confidence is an attempt to expand the subject matter of picture book creation, and it is also an exploration to broaden the channels of traditional cultural transmission. As an intangible cultural heritage, Huai salt drying technology has a wide variety, diverse forms, and a long history. It is an important part of the traditional handicrafts of the Chinese nation [4].

\subsection{Design Ideas}

\subsubsection{References and Excellent Cases of Huai Salt Drying Technology}

The picture book design of Huai salt drying technology not only needs to rely on its own cultural heritage, but also needs to learn from outstanding works, and take the essence and remove the dross to carry out the creative design of the picture book. For example, Zhang Zhiqi's book "Shandong Intangible Cultural Heritage Oral Dance History" shows the enthusiasm and excitement of Shandong Yangko Dance. As one of the most down-to-earth intangible cultural heritage, the whole book is full of folk art and the joy of folk life. Each part will be clipped with colorful soft rice paper to render this festive atmosphere. The colorful paper is used to compare the floating ribbons when dancing Yangko, which has a synesthetic effect and arouses strong resonance among readers. The strong colors and the coated paper on the normal inner pages are interspersed with each other, which has a sense of rhythm and melody, enriching the form of the inner pages and also meeting the visual needs of readers. This creative idea combines the rhythmic beauty of music and gives the overall design soul. It is a highlight of its design that readers can feel the rich and joyful atmosphere of Shandong Yangko even if they are not watching the Yangko Dance through the feeling of materials and colors. In the presentation of the book design, the designers should also learn how Zhang Zhiqi controls the material and the color that gives people the feeling. Huai salt drying technology should also be as close as possible to the materials and colors in the selection of materials and colors, and grasp the degree of texture performance. In the design, it is also necessary to pay attention to the integration of multiple art forms to enhance its effect [5].

\subsubsection{Design Practice of Huai Salt Drying Technology}

Creative picture book design has a certain functional value. With the continuous development of the modern technological era, the behavior of reading pictures is also subtly affecting the thinking 
and behavior of contemporary people [6]. Therefore, the spread of picture book forms is becoming more and more important. Huai salt drying technology provides a new design language and material for the field of picture book creative design. Today's picture books are divided into two types: children's picture books and adult picture books. For more targeted research, the type of picture books in this study is adult picture books [7]. In order to fully explore the creative research on picture books made by Huai salt drying technology, and to enhance the visual effects and aesthetic experience of picture book creativity, it is necessary to deeply explore its methods in the field of picture book creativity. This paper mainly explores three aspects of creative ideas, multiple art forms and design elements, as shown in "Figure 2".

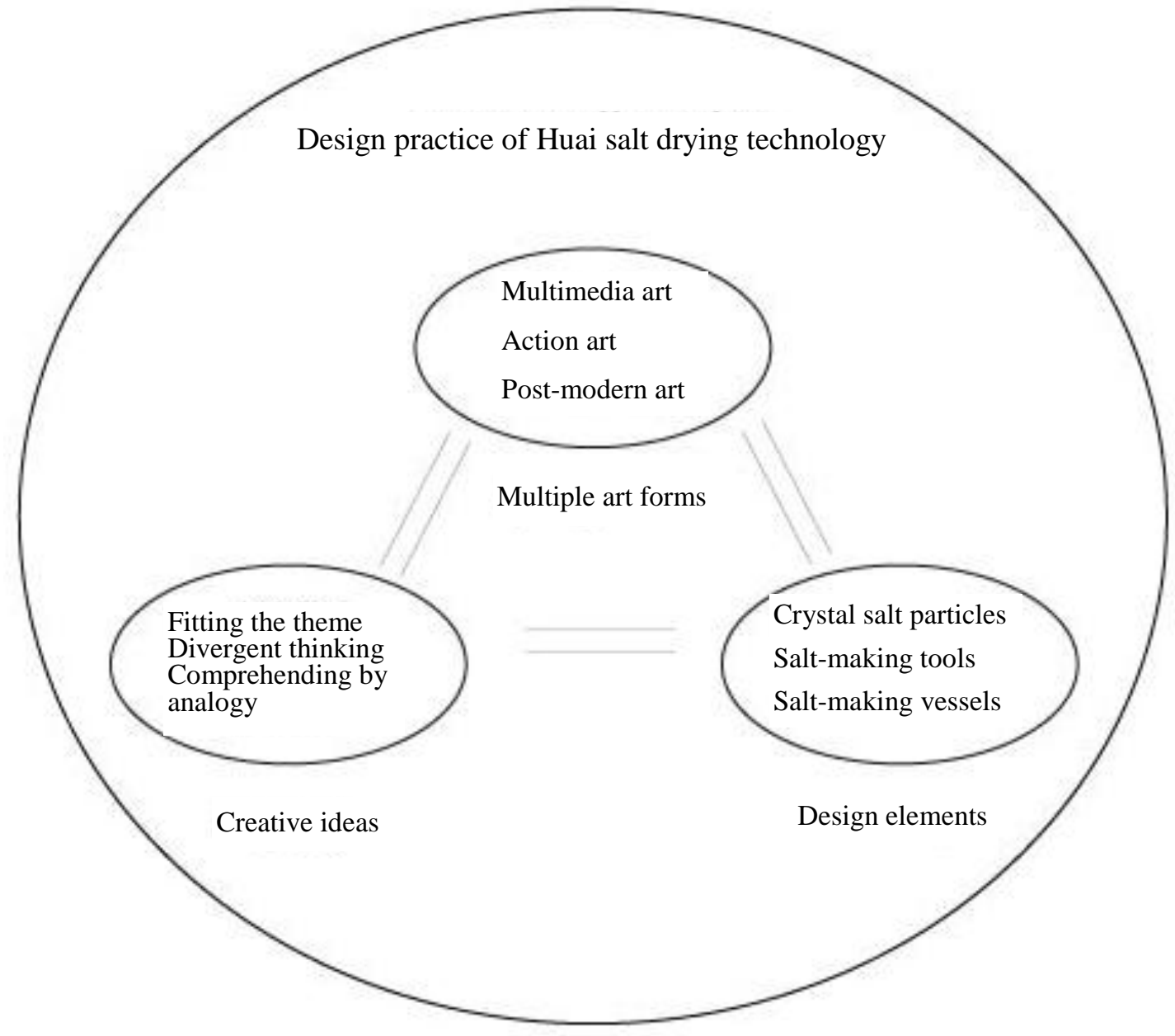

Figure 2 Design and practice of drying technology of Huai salt (self-drawn).

\subsubsection{Creative Ideas}

Because Huai salt drying technology is intangible, it needs to be transformed from intangible to tangible in the design process. In this process, the creative ideas that can diverge are relatively wide. In general, creative ideas need to start designing from the aspects of shape, color, material, typography, cover design, inner page design letter case design, etc. In terms of creative thinking, the designer should follow the principle of restoring and carrying forward the ancient techniques, and express the process of Huai salt drying technology, the texture of Huai salt products, the tools of drying Huai salt, and their changes. For example, the authors' creative design for picture books uses the coastline of the place where Huai salt is produced as the edge of the picture book cover in the shape of the picture book cover design ("Figure 3"). In terms of material selection, hard cardboard with fine texture is used. Dark blue, light blue, gray blue and gray green are used to create a gradual and superimposed effect, and at the same time it is close to the concept of Huai salt by the sea. 
("Figure 4") The technical part of the inner page design uses blue line draft graphics as illustrations, which echoes the main color of the entire book. The vertical typesetting of ancient books is used to present the ancient feeling through the form of thread installation, and some modern elements are added. Through the combination of ancient and modern feelings, a new form of expression of the inheritance of technology is created.

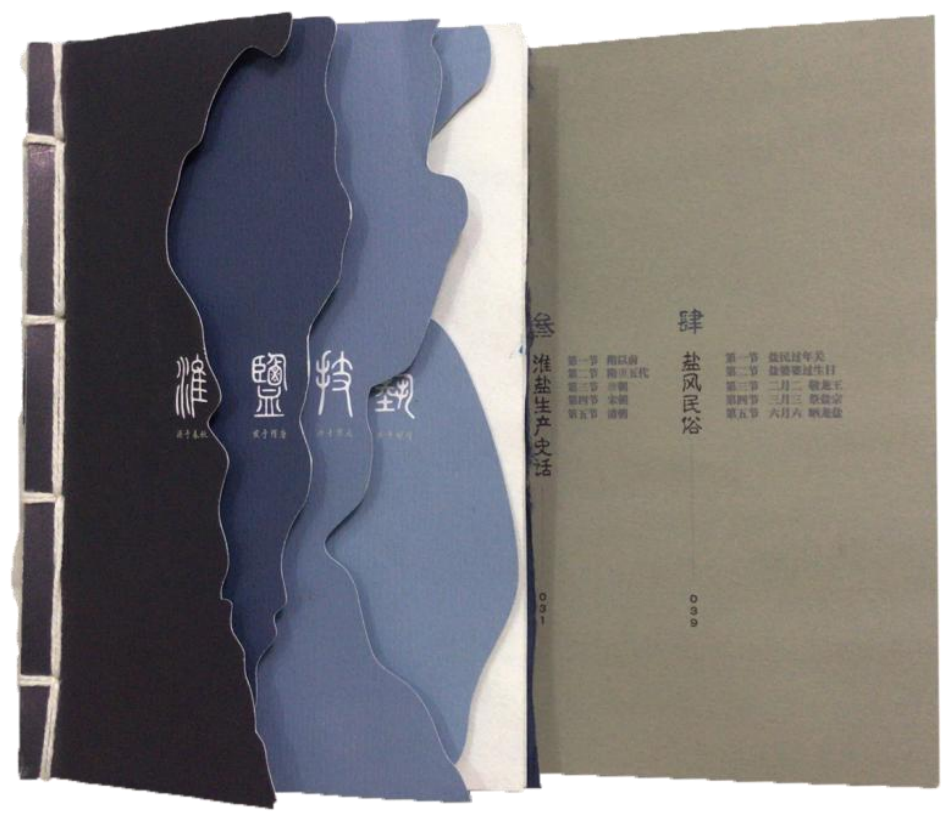

Figure 3 Picture book cover design (self-drawn).

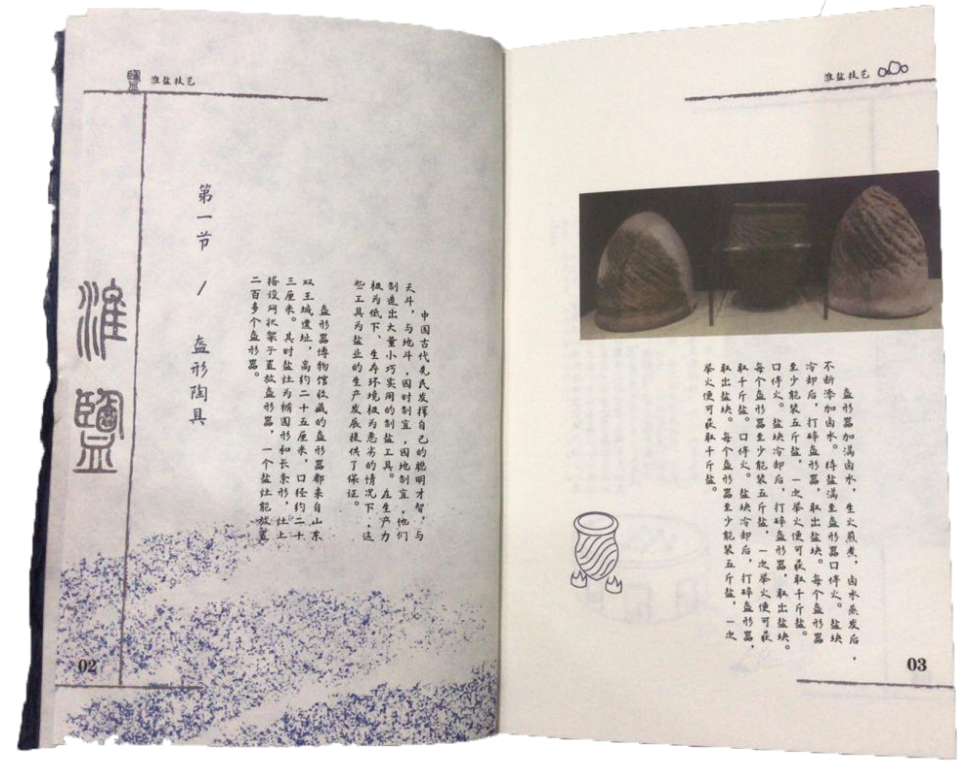

Figure 4 Inside design (self-drawn).

\subsubsection{Various Art Forms}

As a picture book of art, it is essentially the expression and continuation of an action. Therefore, the form of expressing actions in picture books is also indispensable. Digital media art has become an indispensable part of the modern era [8]. Designers can borrow multimedia art to perform artificial 
intelligence, augmented reality and other technologies to restore and reproduce the scenes of drying Huai salt, which provides a better method for the understanding of knowledge and even transmission. Performance art is a new art form that breaks the traditional art. Designers can set up performance art links when designing picture books, increase interaction with readers, make readers more experience, and quickly understand the basic content of Huai salt drying technology. In the design, the authors draw the attention of readers by designing the small opening page. Readers can read the small opening page together when turning to the page to increase the interaction with the readers, thereby inspiring their interest in reading and achieving the spread of skills.

\subsubsection{Design Elements}

The final design elements in the Huai salt drying technique are the salt particles themselves and the constantly changing salt-making tools
("Figure 5" and "Figure 6"). The whole body is crystal white and the body is hard. Because Huai salt is granular, readers need a certain sense of synesthesia during the touch process to satisfy their sense of experience. Therefore, the tactile experience should be paid attention to in the design of picture books. The authors borrowed Cuban paper and other paper with uneven texture in the design. The authors use blue Cuban paper to adhere to the front and back of ordinary paper. The front side is manually torn into the shape of a map of Jiangsu Province. Combining the design form of dots, lines and surfaces, the white Cuban paper is torn into particles to simulate the passage of Huai salt. The form of the coarse salt formed after extraction is used to form a complete layout with discarded white strings. The illustration design in the inner page should be integrated after reference to the original literature, such as the salt-leaching technique in the "Picture of Boiling Wave" ("Figure 7"), etc.

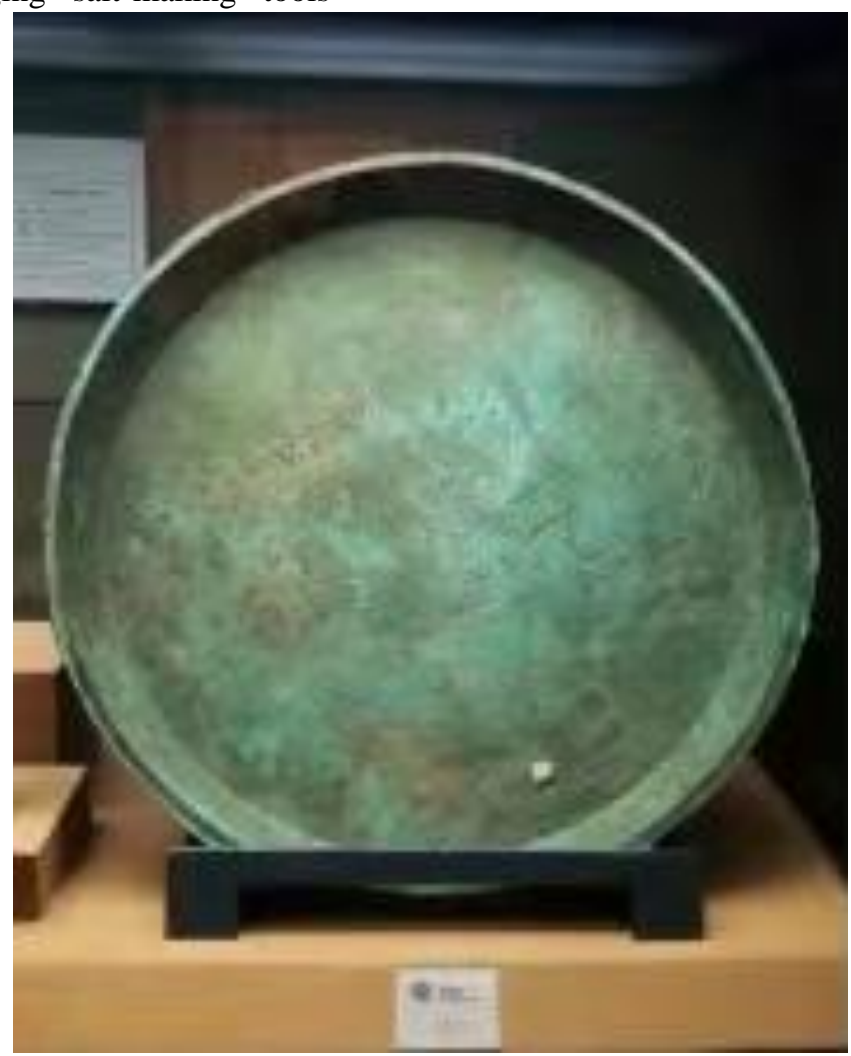

Figure 5 Pan Pan (from Internet). 


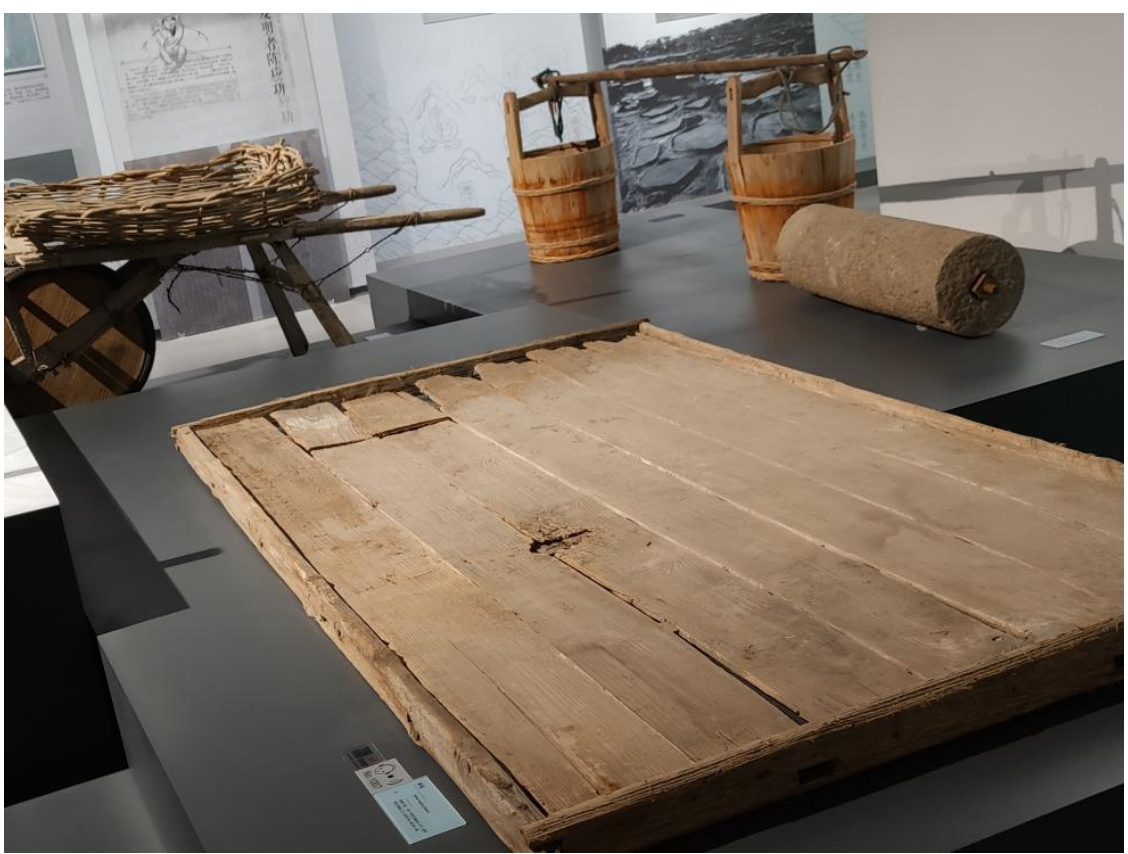

Figure 6 Drying board (self-photographed).

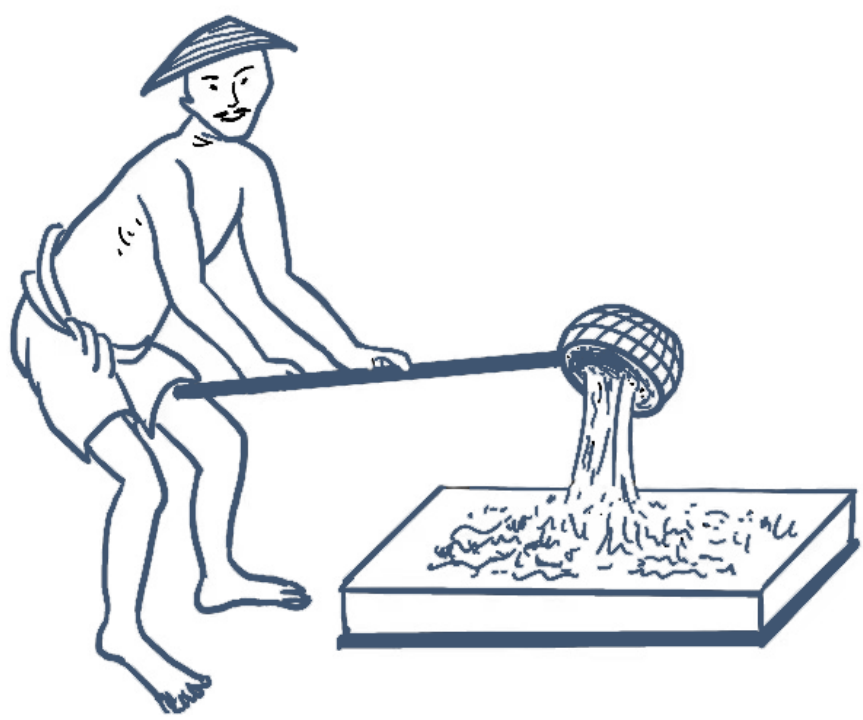

Figure 7 Illustration design (self-drawn).

\section{CONCLUSION}

In the course of development, the skills, tools, and production processes handed down over thousands of years can become excellent painting materials, turning it and its books into research objects, creating and recreating them, to form a picture book design, which can make them propaganda and disseminate among a wide range of reading groups, combine the two organically, and improve the efficiency of communication. In simple terms, a picture book is a combination of pictures and words to interpret a story with a plot. It is a book that expresses the emotion of the story and a special theme. In the process of designing picture books, the factors of art, aesthetics, fine arts, literature and other dimensions are combined. Designers need to fully understand all aspects of 
Huai salt drying technology. Therefore, in the production process, designers have been put forward higher comprehensive requirements. They need to pay attention to readers' aesthetic and reading needs, and pay attention to the development trend and changing trend of the aesthetics of the times. The $\mathrm{Z}$ era emphasizes the uniqueness and diversified expressions of aesthetic forms. Creative picture books are a carrier for inheriting and carrying forward intangible heritage techniques, and they can be appropriately used and displayed on ancient utensils, such as pots and pans. The use of artistic expression of works of art to promote traditional culture and inherit traditional handicrafts adds to the fun of inheritance.

\section{REFERENCES}

[1] Ren Yuan, Qin Huiyou, Research on the Experiential Binding Design of "Yangzhou Paper-cut" [J]. West Leather, 2021, 43(02): 41-42. (in Chinese)

[2] Lu Tonggen, Huai Salt Elements in Lianyungang Regional Culture [J]. Jiangsu Local Chronicles, 2019(04): 12-14. (in Chinese)

[3] Sun Jiakuan, Inheriting the Drying Skills Is the Responsibility of the New Generation of Craftsmen [J]. China Salt Industry, 2017(24): 77-78. (in Chinese)

[4] Zeng Zhu, Inheritance and Innovation of Traditional Culture in the Teaching of Film and Television Animation — Taking Jingweier Culture Series Picture Book Creation Project as an Example [J]. Home Drama, 2021(15): 169-170. (in Chinese)

[5] Feng Beibei, Diversified Forms of Book Binding from Technology Beauty Perspective [J]. Packaging Engineering, 2017, 38(06): 213-218. (in Chinese)

[6] Wang Xiaodan, Research on Book Design Involved in Text Creation: Taking Zhu Yingchun's Creative Book Design Practice as an Example [J/OL]. Science-Technology \& Publication, 1-7 [2021-06-14]. (in Chinese)

[7] Bin Youyan, Research on the Design of Adult Picture Books [D]. Kunming University of Science and Technology, 2014. (in Chinese)
[8] Yan Li, Application Research of Traditional Craft in Handmade Book Art [J]. Packaging Engineering, 2017, 38(08): 76-79. (in Chinese) 